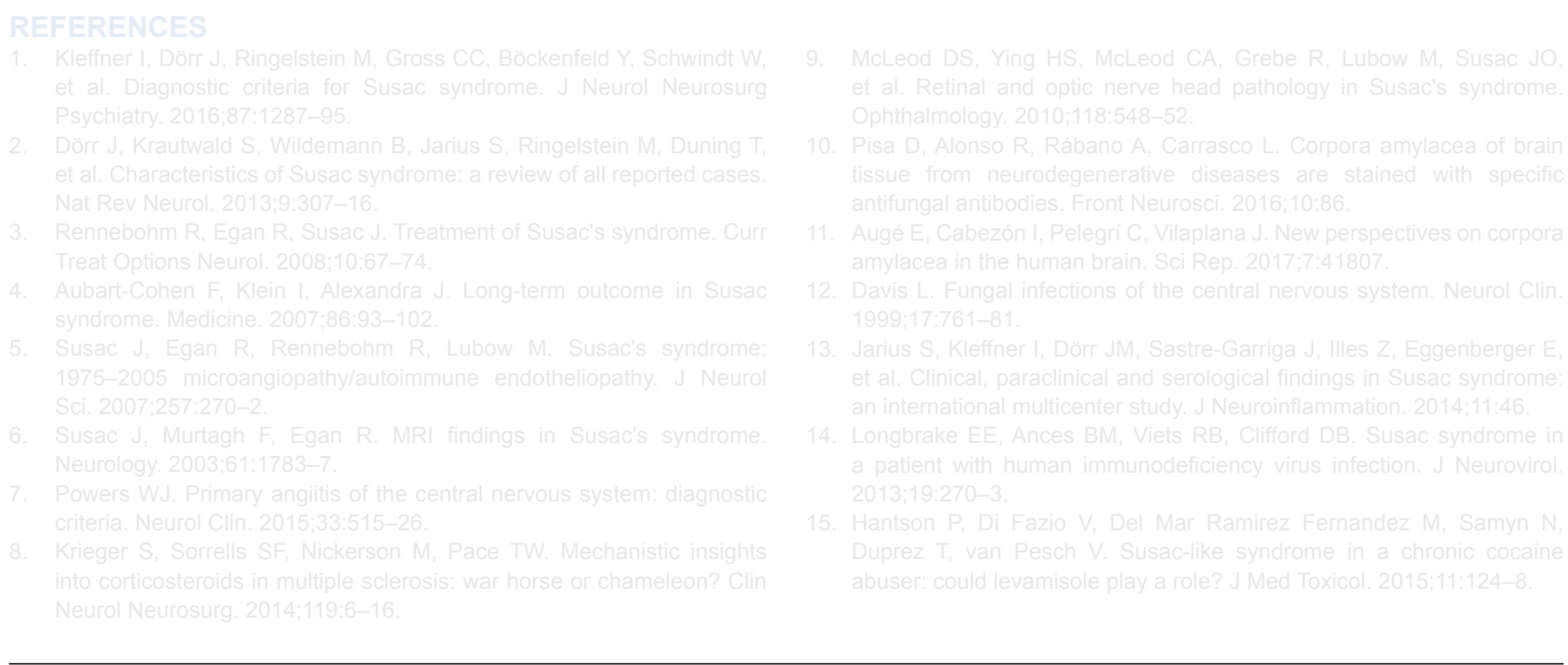

\title{
Hemoperitoneu Espontâneo na Gravidez
}

\section{Spontaneous Hemoperitoneum in Pregnancy}

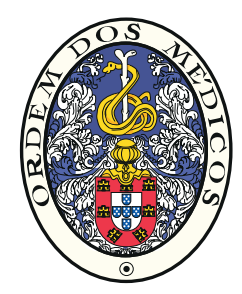

Daniela GONÇALVES $₫ 1$, Marcília TEIXEIRA², Rosa RODRIGUES ${ }^{3}$, Jorge BRAGA ${ }^{3}$
Acta Med Port 2019 Nov;32(11):730-732 - https://doi.org/10.20344/amp.11169

\section{RESUMO}

A endometriose é uma doença benigna, crónica e estrogénio-dependente. Pensa-se que a gravidez tenha um efeito positivo na endometriose através da inibição da ovulação, contudo tem surgido evidência da associação da endometriose não só com infertilidade, mas também com complicações obstétricas. Descreve-se o caso de uma grávida de 34 semanas, com antecedentes clínicos e ecográficos sugestivos de endometriose, admitida por dor abdominal súbita e intensa. Durante a cesariana emergente, realizada por bradicardia fetal sustentada, constatou-se a presença de um volumoso hemoperitoneu e focos hemorrágicos na parede uterina posterior. Apesar de raro, o hemoperitoneu espontâneo é uma complicação possível na gravidez, sobretudo em mulheres com antecedentes de endometriose. Dadas as complicações materno-fetais associadas, uma rápida suspeição diagnóstica e intervenção são fundamentais.

Palavras-chave: Complicações na Gravidez; Endometriose; Gravidez; Hemoperitoneu

\section{ABSTRACT}

Endometriosis is a benign, estrogen-dependent chronic disorder. Pregnancy is considered to have a positive effect on endometriosis due to blockage of ovulation; however, evidence is emerging on the role of endometriosis not only in infertility but also in poor pregnancy outcomes. We present the case of a pregnant woman admitted for sudden and severe abdominal pain at 34 weeks gestation. Her previous medical history included endometriosis suspected by clinical symptoms and ultrasound. During cesarean section, performed by sustained fetal bradycardia, a large volume hemoperitoneum and multiple hemorrhagic foci in the posterior uterine wall were detected. Although rare, spontaneous hemoperitoneum may occur in pregnancy, especially in women with endometriosis. Thus, a prompt suspicion and expedite intervention are needed to improve maternal and fetal outcomes.

Keywords: Endometriosis; Hemoperitoneum; Pregnancy; Pregnancy Complications

\section{INTRODUÇÃO}

A endometriose define-se pela presença de tecido endometrial fora do útero, mais frequentemente na pelve, afetando cerca de $10 \%$ das mulheres em idade reprodutiva. ${ }^{1}$ Clinicamente pode associar-se a dor pélvica, dismenorreia grave, dispareunia e infertilidade, ou ser assintomática e descoberta de forma acidental intra-operatoriamente. ${ }^{2}$
É historicamente aceite que a gravidez tem um efeito positivo na endometriose e nos seus sintomas devido ao estado anovulatório, que previne a hemorragia nos implantes, e às alterações metabólicas, hormonais, imunológicas e angiogénicas que caracterizam a gravidez. ${ }^{2}$ Contudo, têm sido reportadas complicações obstétricas associadas à endometriose, como o hemoperitoneu espontâneo (HE) que apesar de raro pode pôr em risco a vida materna e fetal. ${ }^{3-5}$

1. Serviço de Obstetrícia e Ginecologia. Centro Materno-Infantil do Norte. Centro Hospitalar do Porto. Porto. Portugal.

2. Serviço de Ginecologia. Centro Materno-Infantil do Norte. Centro Hospitalar do Porto. Porto. Portugal.

3. Serviço de Obstetrícia. Centro Materno-Infantil do Norte. Centro Hospitalar do Porto. Porto. Portugal.

$\triangle$ Autor correspondente: Daniela Gonçalves. danielareisgoncalves@hotmail.com

Recebido: 05 de novembro de 2018 - 15 de março de 2019 | Copyright @ Ordem dos Médicos 2019 


\section{CASO CLÍNICO}

Apresentamos o caso clínico de uma mulher de 32 anos, sem patologia médica conhecida, nuligesta, com queixas de dismenorreia intensa e incapacitante desde os 18 anos. Aos 24 anos iniciou contracetivo oral combinado contínuo com melhoria clínica significativa. Apresentava, desde há sete anos, imagem ecográfica sugestiva de endometrioma do ovário esquerdo com cerca de $4 \mathrm{~cm}$, mantendo dimensões estáveis. Ao exame ginecológico era palpável uma formação nodular retrocervical, com cerca de $2 \mathrm{~cm}$. Engravidou espontaneamente um ano e meio após suspender contraceção. Durante a gravidez apenas há referência à tumefação anexial na ecografia do primeiro trimestre, mantendo características e dimensões sobreponíveis.

Às 34 semanas de gestação recorreu ao serviço de urgência por dor abdominal generalizada, constante, de intensidade moderada e início súbito durante a aula de preparação para o parto. Sem outras queixas associadas. Apresentava pele e mucosas pálidas e encontrava-se hemodinamicamente estável. O abdómen era doloroso à palpação global mas sem sinais de irritação peritoneal. Não apresentava perda vaginal de sangue ou líquido amniótico e o colo encontrava-se formado e fechado. A ecografia revelou um feto com boa vitalidade, placenta sem áreas aparentes de descolamento e líquido amniótico normal. A cardiotocografia registava contractilidade uterina regular, pelo que se manteve vigilância. Três horas após verificou-se um agravamento da dor abdominal com sinais de irritação peritoneal e hipertonia uterina. Detetou-se ecograficamente uma bradicardia fetal sustentada, decidindo-se cesariana emergente por suspeita de descolamento prematuro da placenta com hemorragia oculta.

Intra-operatoriamente constatou-se a presença de um volumoso hemoperitoneu com coágulos abundantes. Foi extraido um feto com $2345 \mathrm{~g}$ e índice de Apgar 4/6/6. O líquido amniótico apresentava características normais.

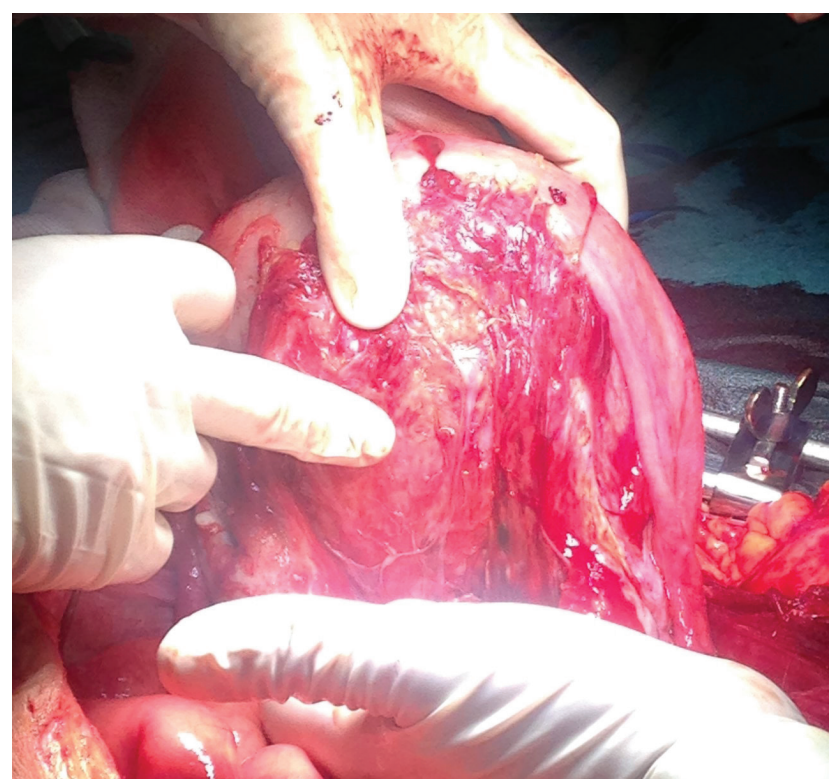

Figura 1 - Parede uterina posterior despolida com focos hemorrágicos ativos
Durante a revisão da cavidade abdominal, em colaboração com a Cirurgia Geral, verificou-se que a parede uterina posterior apresentava aspeto despolido, com hemorragia ativa em toalha, sobretudo à direita, sugerindo rotura de varicosidades e aderências prévias (Fig. 1). Os anexos encontravam-se aderentes à face posterior do útero, sobretudo à esquerda (Fig. 2). A hemostase foi efetuada com Vicryl $2 / 0$, electrocoagulação e colocação de hemostático absorvível em placas sobre a parede uterina posterior. Durante a exploração da cavidade abdominal ocorreu uma lesão iatrogénica do baço com necessidade de esplenectomia para hemostase.

A puérpera e o recém-nascido apresentaram boa evolução clinica tendo alta ao nono e $14^{\circ}$ dia pós-operatório, respetivamente. Em ressonância magnética pélvica, realizada 16 meses após a cirurgia, é reportado um espessamento dos ligamentos útero-sagrados e da reflexão peritoneal posterior, condicionando repuxamento medial dos ovários sobretudo à esquerda, e uma área linear de hipossinal que se estende da vertente posterior do útero à vertente externa do colon sigmoide, sugerindo endometriose profunda.

\section{DISCUSSÃO}

A dor abdominal é uma queixa comum e frequentemente benigna na gravidez, contudo, é essencial identificar os casos cuja etiologia é potencialmente grave, requerendo intervenção urgente. ${ }^{6,7}$ Inicialmente, devem-se excluir as etiologias associadas à gravidez, uma vez que estas têm maior probabilidade de impacto na grávida e no feto. ${ }^{6}$ No caso apresentado, na ausência de elevação tensional de novo, foram colocadas como hipóteses de diagnóstico, inicialmente, trabalho de parto, corroborado pela contratilidade uterina regular, e posteriormente, descolamento placentar e rotura uterina, corroborados pelo agravamento da dor abdominal, instabilidade hemodinâmica, hipertonia uterina e bradicardia fetal, motivando uma intervenção emergente. ${ }^{7}$

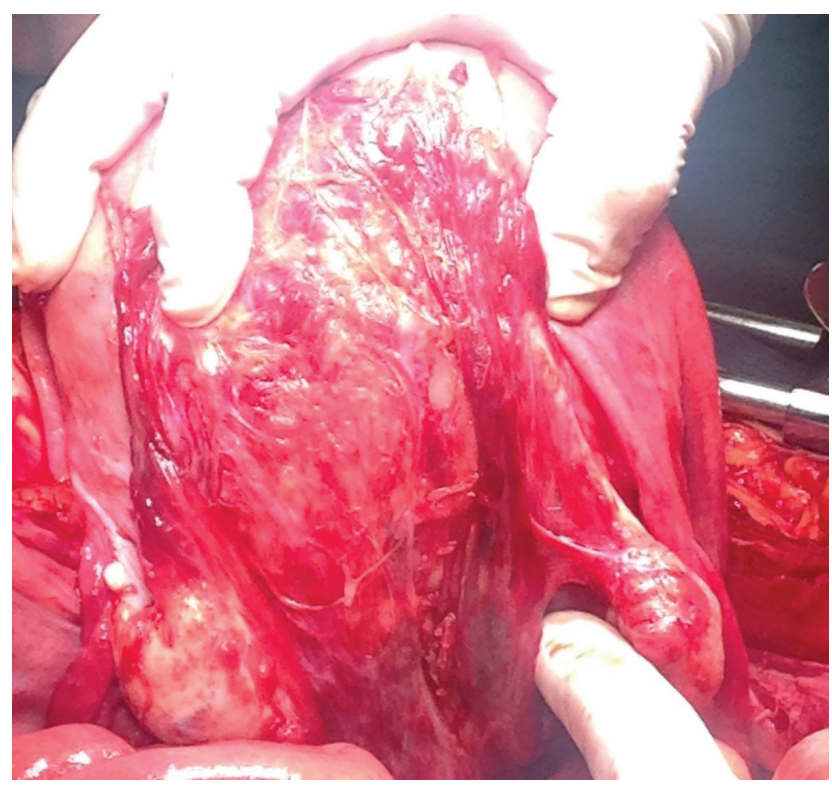

Figura 2 - Anexo esquerdo aderente à face posterior do útero 
A avaliação dos antecedentes pessoais e obstétricos é importante nos casos de dor abdominal na gravidez, podendo orientar a avaliação diagnóstica. ${ }^{6}$ No caso apresentado é relevante a suspeita prévia de endometriose pélvica, uma vez que vários estudos têm reportado complicações na gravidez associadas à endometriose, tais como aumento do volume, rotura e infeção de endometriomas, perfuração intestinal espontânea, apendicite, rotura uterina, rotura uretérica e HE. ${ }^{8}$ Tais complicações são atribuíveis a aderências, ao processo inflamatório crónico dos vasos e tecidos e à invasão da parede de vasos e estruturas pelo tecido endometriótico decidualizado. ${ }^{2}$

O HE durante a gravidez é um evento raro, mas ameaçador da vida materna e fetal. Pode associar-se à rotura do útero, vasos, quistos ováricos ou rotura hepática em casos de pré-eclampsia grave., ${ }^{4,8} \mathrm{~A}$ endometriose é reconhecida como fator de risco major para HE na gravidez, os estudos reportam que mais de $50 \%$ dos casos de HE na gravidez se associam a endometriose. 1,3 $^{1,}$ Trinta e nove casos de HE secundário a endometriose foram identificados na revisão sistemática de Maggiore et al e 33 casos na revisão de Lier et al. ${ }^{8,9} \mathrm{~A}$ hemorragia tem origem mais comum na serosa da parede uterina posterior, ligamentos redondos, útero-sagrados e vasos útero-ováricos, tal como no caso descrito. ${ }^{2,8,10}$ Hemorragias intra-abdominais espontâneas na gravidez têm mais frequentemente origem venosa e o aumento da pressão venosa, provocada por esforços físicos, pode desencadear a rotura de vasos e consequentemente o HE. 2,11,12 Sessenta e nove por cento das grávidas na revisão de Maggiore et al apresentavam clínica de choque hipovolémico à admissão, sendo frequente a associação com dor abdominal e desacelerações cardíacas fetais,

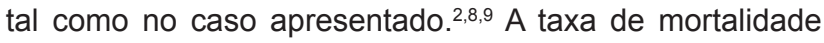
perinatal na literatura é de $27 \%$ e apenas um caso de morte materna foi reportado. ${ }^{9}$

Apesar da evidência crescente de que a endometriose é fator de risco major para a ocorrência de HE na gravidez, não é possível identificar as grávidas em maior risco, nem há evidência de que o tratamento médico ou cirúrgico prévio reduza o risco de complicações. Assim, um elevado

\section{REFERÊNCIAS}

1. Cozzolino M, Corioni S, Maggio L, Sorbi F, Guaschino S, Fambrini M. Endometriosis-related hemoperitoneum in pregnancy: a diagnosis to keep in mind. Ochsner J. 2015;15:262-4.

2. Gao FM, Liu GL. Four case reports of endometriosis-related hemoperitoneum in pregnancy. Chin Med J. 2018;131:502-4.

3. Brosens IA, Lier MC, Mijatovic V, Habiba M, Benagiano G. Severe spontaneous hemoperitoneum in pregnancy may be linked to in vitro fertilization in patients with endometriosis: a systematic review. Fertil Steril. 2016;106:692-703.

4. Vigano P, Corti L, Berlanda N. Beyond infertility: obstetrical and postpartum complications associated with endometriosis and adenomyosis. Fertil Steril. 2015;104:802-12.

5. Leone Roberti Maggiore U, Ferrero S, Mangili G, Bergamini A, Inversetti A, Giorgione V, et al. A systematic review on endometriosis during pregnancy: diagnosis, misdiagnosis, complications and outcomes. Hum Reprod Update. 2016;22:70-103.

6. Kilpatrick C. Approach to acute abdominal pain in pregnant and postpartum women. UpToDate. [consultado 2019 mar 11]. Disponível em: https://www.uptodate.com/contents/approach-to-acute-abdominalpain-in-pregnant-and-postpartum-women. nível de suspeição em grávidas com dor abdominal e antecedentes de endometriose é essencial para o diagnóstico e intervenção precoce nestas situações.

\section{AGRADECIMENTOS}

Os autores gostariam de agradecer aos seguintes colaboradores:

Fernanda Pacheco, Serviço de Obstetrícia, Centro Materno-Infantil do Norte, Centro Hospitalar do Porto, Porto, Portugal. Isabel Pereira, Serviço de Ginecologia, Centro Materno-Infantil do Norte, Centro Hospitalar do Porto, Porto, Portugal. Fernando Correia, Serviço de Anestesia, Centro Hospitalar do Porto, Porto, Portugal. Filomena Pinto, Serviço de Anestesia, Centro Hospitalar do Porto, Porto, Portugal. Ana Novo, Serviço de Neonatologia, Centro Materno-Infantil do Norte, Centro Hospitalar do Porto, Porto, Portugal. Dulce Oliveira, Serviço de Neonatologia, Centro Materno-Infantil do Norte, Centro Hospitalar do Porto, Porto, Portugal. Alexandre Morgado, Serviço de Ginecologia, Centro Materno-Infantil do Norte, Centro Hospitalar do Porto, Porto, Portugal.

\section{PROTEÇÃO DE PESSOAS E ANIMAIS}

Os autores declaram que os procedimentos seguidos estavam de acordo com os regulamentos estabelecidos pelos responsáveis da Comissão de Investigação Clínica e Ética e de acordo com a Declaração de Helsínquia da Associação Médica Mundial.

\section{CONFIDENCIALIDADE DOS DADOS}

Os autores declaram ter seguido os protocolos do seu centro de trabalho acerca da publicação dos dados de doentes.

\section{CONFLITOS DE INTERESSE}

Não existem conflitos de interesse a declarar.

\section{FONTES DE FINANCIAMENTO}

Não foi recebido qualquer subsídio ou bolsa.

7. Koshy Zachariah S, Fenn M, Jacob K, Alias Arthungal S, Anna Zachariah S. Management of acute abdomen in pregnancy: current perspectives. Int J Womens Health. 2019;11:119-34.

8. Leone Roberti Maggiore $U$, Inversetti $A$, Schimberni $M$, Viganò $P$, Giorgione V, Candiani M. Obstetrical complications of endometriosis, particularly deep endometriosis. Fertil Steril. 2017;108:895-912.

9. Lier MC, Malik RF, Ket JC, Lambalk CB, Brosens IA, Mijatovic V. Spontaneous hemoperitoneum in pregnancy (SHiP) and endometriosis - a systematic review of the recent literature. Eur J Obstet Gynecol Reprod Biol. 2017;219:57-65.

10. Lier M, Malik RF, van Waesberghe J, Maas JW, van Rumpt-van de Geest DA, Coppus SF, et al. Spontaneous haemoperitoneum in pregnancy and endometriosis: a case series. BJOG. 2017;124:306-12.

11. Hodgkinson $\mathrm{CP}$, Christensen RC. Hemorrhage from ruptured uteroovarian veins during pregnancy; report of 3 cases and review of the literature. Am J Obstet Gynecol. 1950;59:1112-7.

12. Brosens IA, Fusi L, Brosens JJ. Endometriosis is a risk factor for spontaneous hemoperitoneum during pregnancy. Fertil Steril. 2009;92:1243-5. 